\title{
NOISE-INDUCED STATISTICALLY STABLE OSCILLATIONS IN A DETERMINISTICALLY DIVERGENT NONLINEAR DYNAMICAL SYSTEM*
}

\author{
KATARÍNA BOĎOVÁ ${ }^{\dagger}$ AND CHARLES R. DOERING
}

To Dave Levermore on his 60th birthday with friendship and appreciation

\begin{abstract}
Inspired by partial differential equation models of homogeneous convection possessing heteroclinic connections to infinity, we study a two dimensional system of ordinary differential equations whose solutions diverge exponentially for almost all initial conditions. Random perturbations of the dynamical system destabilize the divergences resulting in stochastic oscillations. Stochastic Lyapunov function methods are used to prove the existence of a statistically stationary state. A novel Monte-Carlo method is implemented to measure the extreme statistics associated with the stochastic oscillations, and a WKB analysis at low noise amplitude is carried out to corroborate the simulations.
\end{abstract}

Key words. Nonlinear dynamical systems, stochastic dynamical systems, Monte-Carlo methods, low-noise asymptotic analysis.

AMS subject classifications. 60H10, 60H30, 60G40, 65C05.

\section{Introduction}

Noise-induced dynamical phenomena are observed in mathematical models arising from applications in physics, chemistry, biology [9], and neuroscience [8]. In some cases a dynamical system's variability arises from underlying discreteness. One such example is an individual-based predator-prey system [12] where it was demonstrated that stochastic cycles are present in the population-level models even though the continuous (infinite population) model fails to display oscillations. Low level noise may also induce oscillating behavior in problems close to a bifurcation, e.g., close to a saddle-node [18] or sniper [7] bifurcation, and in excitable systems [13]. Even though intrinsic internal or externally imposed noise is an integral component of many processes, numerical simulations of theoretically deterministic models may be sensitive to round-off errors that serve as small (albeit artificial) random perturbations.

There is evidence that numerical errors can systematically impact computational simulations of some problems. Recently, Calzavarini et al. [3] studied a model of "homogeneous" Rayleigh-Bénard convection described by nonlinear partial differential equations with spatially periodic boundary conditions. That model contains exact exponentially growing solutions, i.e., heteroclinic connections to infinity, and these solutions accurately fit numerical simulations for a finite time, followed by an unexpected sudden collapse. This pattern is repeated with collapses occurring at seemingly random times even though there is no inherent randomness in the model or in the numerical method. The only systematic error present is the small round-off noise. This example inspired us to look for a simple dynamical system with the same property, i.e., a deterministic model that blows up exponentially for almost all initial conditions

\footnotetext{
*Received: December 28, 2010; accepted (in revised version): May 19, 2011.

${ }^{\dagger}$ Department of Applied Mathematics \& Statistics, Faculty of Mathematics, Physics and Informatics, Comenius University, 84248 Bratislava, Slovakia (bodova@fmph.uniba.sk).

${ }^{\ddagger}$ Department of Mathematics, University of Michigan, Ann Arbor, MI 48109-1043, USA. Department of Physics and Michigan Center for Theoretical Physics, University of Michigan, Ann Arbor, MI 48109-1040, USA and Center for the Study of Complex Systems, University of Michigan, Ann Arbor, MI 48109-1107, USA (doering@umich.edu).
} 
but remains bounded for arbitrarily small stochastic perturbation. The aim here is to reveal some of the dynamical elements responsible for the behavior as well as to explore the limits of rigorous, asymptotic, and numerical analyses of such systems.

Although the role of noise in ordinary differential equations is still not fully understood, some particular questions have been answered already. In one dimension, Scheutzow [16] showed that if a deterministic equation has solutions that explode (i.e., go to infinity) in a finite time for some initial condition then it necessarily explodes in a finite time with probability strictly greater than zero when a constant white noise is added. In different work [17] the same author constructed an example of a twodimensional system where deterministic solutions explode in a finite time uniformly for all initial conditions, but with any arbitrarily small level of additive white noise all solutions are nonexplosive with probability one. The construction of the example is extremely complicated and the dynamical mechanisms at work are unclear. That is, it is not straightforward to identify the key properties of the system that lead to observed behavior.

The main goal of this work is to show that there are simple systems in which arbitrarily small random perturbations, such as numerical round-off error, significantly change the qualitative behavior of solutions. We present a planar deterministic dynamical system designed to capture key features of the homogenous convection problem, namely the property that almost every solution diverges to infinity exponentially in time. Note that the system we consider serves only as a prototype of this behavior and it is not derived from the convection problem. On the other hand, a 3D model with similar behavior was systematically derived for a homogeneous thermohaline convection problem directly from the partial differential equations [10]. Here we aim to capture the central qualitative features in a two dimensional model.

Under the addition of a small magnitude Gaussian noise perturbation, the dynamical system studied in this work exhibits statistical oscillations. Using a theory of stochastic Lyapunov functions [11] we rigorously prove that addition of arbitrary small white noise produces a statistically steady stochastic evolution that is a stationary Markov process. Next, we develop an effective numerical method to find the distribution of collapse times that capture random periods of the oscillations and formulate an ansatz for the long-time behavior of its statistics. We use the ansatz to study the time-inhomogeneous non-constant coefficient Fokker-Planck equation for the related first exit time problem in the long-time limit. Finally, we perform an asymptotic analysis of a corresponding Sturm-Liouville problem and confirm that our numerical and analytical results are consistent.

\section{Model equations}

We study the nonlinear dynamical system

$$
\begin{aligned}
& \frac{d x}{d t}=-x\left(4-x y+x^{2}\right), \\
& \frac{d y}{d t}=\frac{y-x^{3}}{1+x^{2}}
\end{aligned}
$$

with the symmetry $(x, y) \rightarrow(-x,-y)$. The model is designed to have solutions that grow exponentially in time and that are also sensitive to small random perturbations. The system has three equilibria: a saddle $(0,0)$ and two spiral sources $\left(x^{*}, y^{*}\right)=$ $\pm\left(\frac{1}{2}(1+\sqrt{17})^{1 / 2}, \frac{1}{2}(1+\sqrt{17})^{3 / 2}\right)$. There are separatrices from the sources to $\pm \infty$, and also from $\pm \infty$ to the origin. All trajectories eventually escape to infinity along 

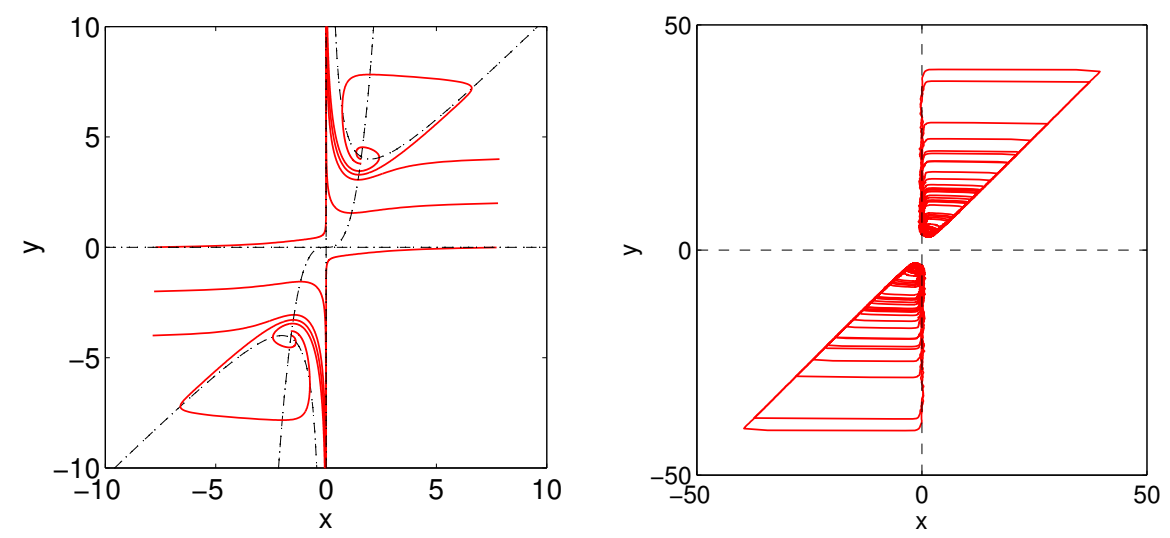

FIG. 2.1. (left) Sample trajectories of the deterministic model (2.1)-(2.2). The $d x / d t=0$ nullclines $4-x y+x^{2}=0$ and $x=0$ and the $d y / d t$ nullcline $y=x^{3}$ are shown as well. (right) Solutions of (2.3)-(2.4) with an added white noise of magnitude $\sigma=1$. Trajectories grow along the $y$-axis before noise pushed them across the approaching $4-x y+x^{2}=0$ nullcline and the trajectory returns back to a neighborhood of the origin. Note the change of vertical scale on the right panel.

the $y$-axis at an exponential rate except for those starting precisely at the fixed points or on the separatrices to (stable manifold of) the origin; see the left panel of Figure 2.1.

The $d x / d t=0$ nullcline $y=x+4 / x$ rapidly approaches the $y$-axis to form a shrinking stability boundary on one side of the attracting trajectory $x(t)=0, y(t)=y_{0} e^{t}$. Trajectories near the $y$-axis thus become very sensitive to small perturbations in the $x$-direction, and in response they may cross the $d x / d t=0$ nullcline and rapidly move away from the $y$-axis before collapsing exponentially towards the origin. Adding Gaussian white noise to the $x$-variable changes the system (2.1)-(2.2) to

$$
\begin{aligned}
d x & =-x\left(4-x y+x^{2}\right) d t+\sigma d W, \\
d y & =\frac{y-x^{3}}{1+x^{2}} d t .
\end{aligned}
$$

As shown in the right panel of Figure 2.1 and in the time-series in Figure 2.3, the noise-perturbed system has several different significant types of dynamics:

- noise-driven (slow) dynamics near the origin;

- growth along the $y$-axis while $4-x y+x^{2}>0$ for $x>0$ (and symetrically for $x<0$ );

- noise-induced transition across the nullcline $y=x+4 / x$;

- rapid drift-dominated flow to large $|x|$ and subsequent exponential collapse to a neighborhood of origin along $y \approx x$.

The shrinking basin of attraction of the attracting trajectory along the $y$-axis together with the collapses towards the origin are the key dynamical properties responsible for the noise-induced phenomena in the model (see Remark 2.2 below). In particular, the deterministic system (2.1)-(2.2) has a resetting property: any trajectory starting from $4-x y+x^{2}<0$ for $x>0$ (and by symmetry for $x<0$ ), i.e., outside of a narrow "half-funnel" around the $y$-axis, will eventually (in finite time) enter a fixed neighborhood of the origin. In terms of the regions $\mathcal{A}_{1}, \mathcal{A}_{2}, \mathcal{B}$, and $\mathcal{R}$ (see Figure 2.2) defined as 
- $\mathcal{A}_{1}=\left\{(x, y): \frac{d|x|}{d t}(x, y)>0,|x| \leq\left|x^{*}\right|\right\}$ (increasing $\left.|x| \leq\left|x^{*}\right|\right)$,

- $\mathcal{A}_{2}=\left\{(x, y): \frac{d|x|}{d t}(x, y)>0,|x| \geq\left|x^{*}\right|\right\}$ (increasing $\left.|x| \leq\left|x^{*}\right|\right)$,

- $\mathcal{B}=\left\{(x, y): \frac{d|x|}{d t}(x, y)<0\right\} \cap\{(x, y): x(y-x)>0\}$ (decreasing $\left.|x|\right)$,

- $\mathcal{R}=\left\{|x| \leq\left|x^{*}\right|,|y| \leq\left|y^{*}\right|\right\}$,

the property reads as follows: any trajectory of (2.1)-(2.2) starting in region $\mathcal{A}_{1}$ eventually enters regions $\mathcal{A}_{2}, \mathcal{B}$ and $\mathcal{R}$ in the order $\mathcal{A}_{1} \rightarrow \mathcal{A}_{2} \rightarrow \mathcal{B} \rightarrow \mathcal{R}$. This reflects the dynamical properties of (2.1)-(2.2) near the one-sided funnel, i.e., near the part of $\mathcal{B}$ along the vertical axis, that drives the trajectories from $\mathcal{A}_{1}$ to $\mathcal{A}_{2}$ in a finite time due to the positive $x$-derivative that is bounded away from zero. The trajectory is consequently driven to the narrow part of region $\mathcal{B}$ along $y=x$ and along this region it enters $\mathcal{R}$ in a finite time. For more details, see [2].

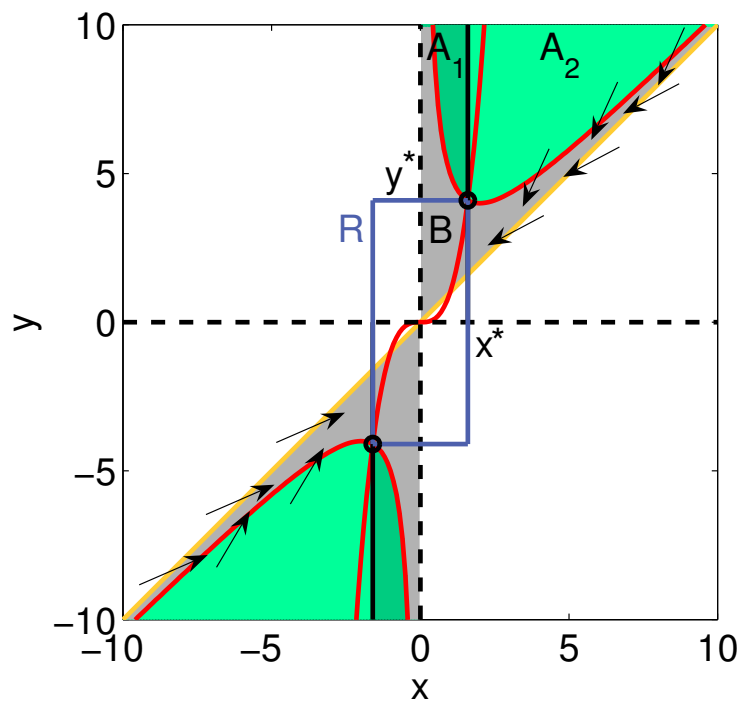

FIG. 2.2. Regions $\mathcal{A}=\mathcal{A}_{1} \cup \mathcal{A}_{2}, \mathcal{B}, \mathcal{R}$. A trajectory of (2.1)-(2.2) starting in $\mathcal{A}_{1}$ enters $\mathcal{A}_{2}$, then $\mathcal{B}$, then $\mathcal{R}$ in a finite time. The red curves correspond to the nullclines $y=x^{3}$ and $y=x+4 / x$ and the black circle is the equilibrium of the system.

REMARK 2.1. Although the system analyzed in this paper has a very particular form, the results obtained can be generalized to a wider class of problems. A straightforward generalization is a nondimensionalized system

$$
\begin{aligned}
& d x=-x\left(4-x y+a x^{2}\right) d t+\sigma d W, \\
& d y=\delta\left(1+x_{0}^{2}\right) \frac{y-b x^{3}}{1+x^{2}} d t,
\end{aligned}
$$

where $\left(x^{*}, y^{*}\right)= \pm\left(x_{0}, b x_{0}^{3}\right)$ are the nontrivial equilibria and $a, b, \delta$ are positive. Note that the system (2.5)-(2.6) is topologically equivalent to (2.3)-(2.4) only in a certain parameter regime. Particularly, there is a restriction on the parameter $\delta$ in terms of $a$ and $b$. The time scale separation constant $\delta$ must be bounded both from below and above. An important role is played by the natural time scale $T^{*}=\left|4-a x_{0}^{2}\right|$ of the system, associated with the nontrivial equilibria $\left(x^{*}, y^{*}\right)$. If $4-a x_{0}^{2}>0$, the bound 


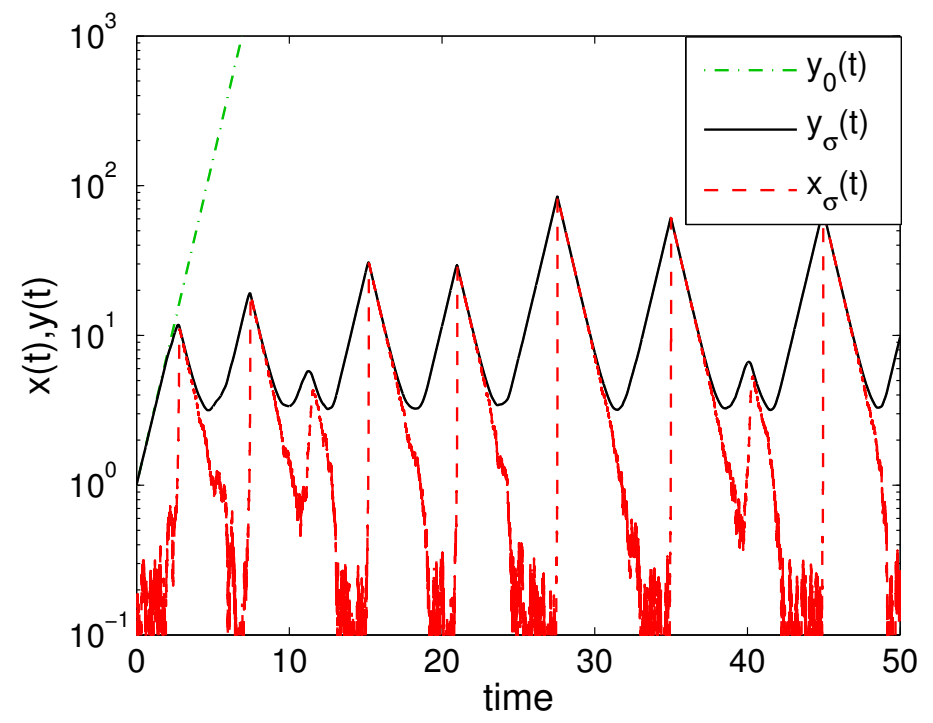

FIG. 2.3. Sample solution of the stochastic model (2.3)-(2.4) showing statistically steady oscillations in time with the noise strength $\sigma=0.5$. Functions $x_{\sigma}(t)$ and $y_{\sigma}(t)$ are plotted in red/dash and black/solid, respectively. The deterministic solution $y_{0}(t)$ is plotted in green/dash-dot.

is symmetrical with respect to $T^{*}: \delta \in\left(T_{b},\left(T^{*}\right)^{2} / T_{b}\right)$, otherwise $\delta \in\left(T^{*},\left(T^{*}\right)^{2} / T_{b}\right)$, $T_{b}>0$. The stochastic Lyapunov function, introduced in Section 3, can be constructed analogously with $\hat{V}(x, y)=(a x-y)^{2}+y^{2}$.

REMARK 2.2. The system (2.3)-(2.4) is sensitive to small noise in the horizontal direction due to the narrowing (on one side) region of attraction of the exact exponentially growing solution along the $y$-axis. Here we present an example of a system with similar dynamics that does not have a SLF:

$$
\begin{aligned}
& d x=-x(1-x y) d t+\sigma d W, \\
& d y=\left(y-x^{3}\right) d t .
\end{aligned}
$$

The two models differ in the resetting property. While the system (2.3)-(2.4) returns back to a neighborhood of the origin at every collapse, the system (2.7)-(2.8) keeps drifting to infinity along the vertical axis, i.e., repeating collapses do not bring the trajectory closer to the origin. A detailed analysis of this model can be found in [2].

\section{Stochastic Lyapunov function}

The theory of stochastic Lyapunov function (SLF) was developed by R. Khasminskii [11]. Consider a time-homogeneous stochastic differential equation in $\mathbb{R}^{n}$

$$
d x=b(x) d t+\sigma(x) d W, \quad x\left(t_{0}\right)=x_{0},
$$

where $W$ is an $n$-dimensional Wiener process and $\sigma(x)=\left(\sigma_{1}(x), \ldots, \sigma_{n}(x)\right)$ with $b, \sigma_{1}, \ldots, \sigma_{n} \in \mathscr{C}\left(\mathbb{R}^{n}, \mathbb{R}^{n}\right)$. Let $\mathcal{K}$ be a compact subset of $\mathbb{R}^{n}$ and let the coefficients 
in (3.1) satisfy the following growth conditions for some $c \in \mathbb{R}$ for any $x, x_{1}, x_{2} \in \mathcal{K}$ :

$$
\begin{aligned}
& |b(x)|+\sum_{k=1}^{n}\left|\sigma_{k}(x)\right| \leq c(1+|x|), \\
& \left|b\left(x_{2}\right)-b\left(x_{1}\right)\right|+\sum_{k=1}^{n}\left|\sigma_{k}\left(x_{2}\right)-\sigma_{k}\left(x_{1}\right)\right| \leq c\left|x_{2}-x_{1}\right| .
\end{aligned}
$$

Assume that

$$
\text { there exists } x_{0} \in \mathbb{R}^{n} \text { such that } x(t) \text { is regular, }
$$

and there exists an auxiliary function $V \in \mathscr{C}^{2}\left(\mathbb{R}^{n}\right)$ satisfying

$$
\begin{aligned}
& V(x) \geq 0, \\
& \sup _{|x|>R} \mathcal{L} V(x)=-A_{R} \rightarrow-\infty \quad \text { as } \quad R \rightarrow \infty .
\end{aligned}
$$

Here $\mathcal{L}$ is a generator of the stochastic process (3.1):

$$
\mathcal{L}=\sum_{i} b_{i}(x) \cdot \partial_{x_{i}}+\frac{1}{2} \sum_{i, j}\left(\sigma(x) \sigma(x)^{\prime}\right) \partial_{x_{i}} \partial_{x_{j}} .
$$

The theory of R. Khasminskii then implies that the stochastic process (3.1) has an invariant measure, i.e., a solution is a stationary Markov process. Such a function $V(x)$ is called a stochastic Lyapunov function. Now we can formulate the main result of the paper.

\section{The main result}

TheOREM 4.1. For a continuous drift function $b(x), b: \mathbb{R}^{2} \rightarrow \mathbb{R}^{2}$, defined in (2.1)(2.2), the solutions of the system $d x / d t=b(x)$ with $x(0)=x_{0}$ satisfy $\lim _{t \rightarrow \infty}|x(t)|=$ $\pm \infty$ for almost all $x_{0} \in \mathbb{R}^{2}$ but solutions of (3.1) with $\sigma_{1}=(\bar{\sigma}, 0)^{T}$ and $\sigma_{2}=(0,0)^{T}$ form a stationary Markov process for an arbitrary $\bar{\sigma}>0$.

The drift function $b(x)$ is chosen according to (2.3)-(2.4). In order to prove Theorem 4.1 it is necessary to construct the SLF satisfying (3.4)-(3.6) for system (2.3)-(2.4). Note that the deterministic system (2.1)-(2.2) does not have a Lyapunov function because almost all its trajectories blow up exponentially. To construct a SLF for (2.3)-(2.4) for arbitrarily small $\sigma$ in the sense of [11] we need to design this auxiliary function for the noise-perturbed system in such a way that the diffusion term in the generator dominates the rest. However, the diffusion term in the generator $\mathcal{L}$ is proportional to $\sigma^{2}$ which may be arbitrarily small. Thus the form of the SLF must depend on $\sigma$ as well. Trajectories are most sensitive to noise in the narrow "funnel" region of $\mathcal{B}$ between the $y$-axis and $\mathcal{A}$, and thus the key is to construct the SLF in this region. Note that the SLF may be defined arbitrarily on any given compact region without violating (3.4)-(3.6).

Proof. We will prove that the system (2.3)-(2.4) satisfies all conditions given by Theorem 4.1. The corresponding deterministic system trivially satisfies all the requirements, having non-diverging solutions only when starting at one of the three fixed points or two stable connections from $\pm \infty$ to the origin. We construct a simple stochastic Lyapunov function that respects the structure of the problem (symmetry, fast/slow phase). The construction consists of three steps. 
(a) Fast region: $\hat{V}(x, y)=(x-y)^{2}+y^{2}$ satisfies SLF condition on $\Omega_{F}=\{(x, y) \in$ $\left.\mathbb{R}^{2}:|x| \geq \bar{C},|y| \geq 1\right\}$, where $\bar{C}=2$.

(b) Slow region: $f(x, \sigma) \hat{V}(x, y)$ satisfies SLF condition on $\Omega_{S}=\left\{(x, y) \in \mathbb{R}^{2}:|x| \leq\right.$ $\bar{C}\}$. In addition, it can be patched with constant multiples of $\hat{V}(x, y)$ on $\Omega_{F}$ so that the result will be $\mathscr{C}^{2}$. The SLF will be defined separately for $y>1$ and by parity for $y<-1$. It will depend on the noise strength $\sigma$.

(c) All the remaining "rough" parts of the function $(|y| \leq 1)$ will be patched together to produce an explicit $\mathscr{C}^{2}$ function $V(x, y, \sigma)$ that satisfies the SLF condition on $\mathbb{R}^{2}$ for arbitrarily small noise strength $\sigma$.

(a) Fast region $\Omega_{F}$. Trajectories of (2.3)-(2.4) are not very sensitive to noise in the fast region $\Omega_{F}$. We choose $\hat{V}(x, y)=(x-y)^{2}+y^{2}$ to obtain

$$
\begin{aligned}
\mathcal{L} \hat{V}(x, y)= & \left(\frac{4}{1+x^{2}}-2 x^{2}\right) y^{2}+\left(8 x+4 x^{3}-\frac{2 x+4 x^{3}}{1+x^{2}}\right) y \\
& +\sigma^{2}-8 x^{2}-2 x^{4}+\frac{2 x^{4}}{1+x^{2}}
\end{aligned}
$$

This quadratic function in $y$ is concave down for $|x| \geq \bar{C}$. For technical purposes we analyze two cases: $|x| \in[\bar{C}, M]$, and $|x| \in[M, \infty]$ for sufficiently large $M$. First, observe that if $x \in[\bar{C}, M]$ then simple inequalities imply that

$$
\mathcal{L} \hat{V}(x, y) \leq-y^{2}+18 M^{3}|y|+\sigma^{2}
$$

and $\mathcal{L} \hat{V}(x, y) \rightarrow-\infty$ when $y \rightarrow \infty$, so the condition (3.6) is satisfied. Next we use concavity of $\mathcal{L} \hat{V}(x, y)$ with respect to variable $y$ to find its maximum on the remaining interval $[M, \infty)$ by setting $\frac{\partial \mathcal{L} \hat{V}}{\partial y}\left(x, y_{m}\right)=0$ and solving for $y_{m}$ :

$$
y_{m}=\frac{2 x^{5}+4 x^{3}+3 x}{2 x^{4}+2 x^{2}-4} x \sim x \text { for } x \gg 1 .
$$

The resulting $y_{m}$ can be furthermore substituted into $\mathcal{L} \hat{V}(x, y)$ :

$$
\mathcal{L} \hat{V}\left(x, y_{m}\right)=\frac{-4 x^{8}+O\left(x^{6}\right)}{2 x^{6}+O\left(x^{4}\right)} \sim-2 x^{2} \text { for } x \gg 1 .
$$

This shows that for $|x| \in[M, \infty)$ the function $\mathcal{L} \hat{V}(x, y)$ is bounded from above by a negative polynomial and therefore approaches $-\infty$ as $|\mathbf{x}| \rightarrow \infty$. In fact, $\mathcal{L} \hat{V}(x, y) \rightarrow$ $-\infty$ as $|(x, y)| \rightarrow \infty$ since it is a polynomial in $y$. Therefore any positive multiple of $\hat{V}(x, y)$ satisfies the SLF condition in $\Omega_{F}$.

(b) Slow region $\Omega_{S}$. The dynamics of (2.3)-(2.4) is very sensitive to the noise magnitude $\sigma$ in the slow region $\Omega_{S}=\left\{(x, y) \in \mathbb{R}^{2}:|x| \leq \bar{C}\right\}$. However, the function $\hat{V}(x, y)=(x-y)^{2}+y^{2}$ does not depend on the noise magnitude $\sigma$, and therefore cannot satisfy condition (3.6). In order to modify it we define the SLF on a half-strip region $|x| \leq \bar{C}, y>1$ (and by symmetry for $y<-1$ ) as

$$
V(x, y, \sigma)=f_{\sigma}(x) \hat{V}(x, y)=f_{\sigma}(x)\left[(x-y)^{2}+y^{2}\right],
$$

where $f=f_{\sigma} \in \mathscr{C}^{2}$ depends on $\sigma$. Note that the smoothness requirement for the SLF imposes boundary conditions on $f(x)$ at $x= \pm \bar{C}$ :

$$
f^{\prime \prime}(-2)=f^{\prime \prime}(2)=0 \text { and } f^{\prime}(-2)=f^{\prime}(2)=0,
$$




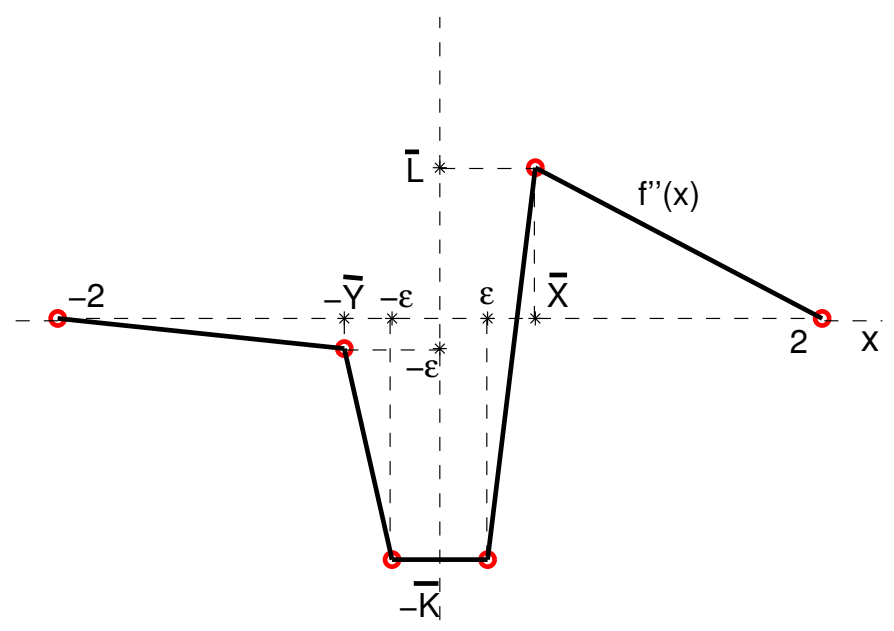

FIG. 4.1. The piecewise defined function $f^{\prime \prime}(x)$ in the slow region. Values of $\bar{K}, \bar{L}, \bar{X}, \bar{Y}$, and $\varepsilon$ depend exclusively on the magnitude of the noise strength $\sigma . \bar{K}$ is chosen big enough and $\varepsilon$ small enough to satisfy the SLF properties.

but also $f(-2), f(2)>0$. The stochastic generator of (2.3)-(2.4) applied to the function $V(x, y, \sigma)$ can be written as

$$
\begin{aligned}
\mathcal{L} V(x, y, \sigma)= & \sum_{n=0}^{3} A_{n}\left(x, f, f^{\prime}, f^{\prime \prime}, \sigma\right) y^{n} \\
= & 2 x^{2} y^{3} f^{\prime}+y^{2}\left(-2 x^{2} f-8 x f^{\prime}-4 x^{3} f^{\prime}+\sigma^{2} f^{\prime \prime}+\frac{4 f}{1+x^{2}}\right) \\
& \quad+y A_{1}\left(x, f, f^{\prime}, f^{\prime \prime}, \sigma\right)+A_{0}\left(x, f, f^{\prime}, f^{\prime \prime}, \sigma\right) .
\end{aligned}
$$

A necessary condition for $V(x, y, \sigma)$ to be a SLF is $y f^{\prime}<0$, i.e., $f^{\prime}<0$ in the half-strip $|x| \leq \bar{C}, y>1$. That implies that $f$ needs to be decreasing in the positive half-strip. Since the system (2.1)-(2.2) blows up exponentially (and for that reason it does not have a LF) it must be the stochastic term in the generator $\frac{1}{2} \sigma^{2} \partial_{x x} V(x, y, \sigma)$ that cancels all the terms that blow up and makes $\mathcal{L} V(x, y, \sigma)$ approach $-\infty$. This leads to the observation that $V(x, y, \sigma)$ must be concave in $x$ for $x \approx 0$ and also that its second derivative (with respect to $x$ ) must dominate positive terms in $\mathcal{L} V(x, y, \sigma)$. The construction is divided into three steps:

(i) Construct a piecewise linear function $f^{\prime \prime}(x)$ that is

- decreasing on $[-2,-\varepsilon]$ and $[\bar{X}, 2]$,

- equal to a large negative constant on $[-\varepsilon, \varepsilon]$,

- increasing on $[\varepsilon, \bar{X}]$,

- has a zero integral,

and depends on parameters $\varepsilon, \bar{K}, \bar{L}, \bar{X}$, and $\bar{Y}$ (and all depend on the noise magnitude $\sigma$ ), as in Figure 4.1. 
(ii) Integrate the function $f^{\prime \prime}(x)$ twice to find $f(x)$ and choose the parameters $\bar{A}, \bar{B}, \bar{L}, \bar{X}, \bar{Y}$ to satisfy $f^{\prime \prime}(-2)=f^{\prime \prime}(2)=f^{\prime}(-2)=f^{\prime}(2)=0, f(-2)=\bar{A}>0$, $f(2)=\bar{B}=\varepsilon>0$ and $f^{\prime}(x)<0$ for $|x|<\bar{C}$.

(iii) Choose the remaining parameter values $\varepsilon$ and $\bar{K}$ so that the condition (3.6) is valid, i.e., that $A_{2}(x)<0$.

(i) The piecewise linear function $f^{\prime \prime}(x)$ is constructed to satisfy conditions in (i). The simplest candidate is schematically plotted on Figure 4.1. There are 5 parameters: $\varepsilon$, $\bar{K}, \bar{L}, \bar{X}$, and $\bar{Y}$ :

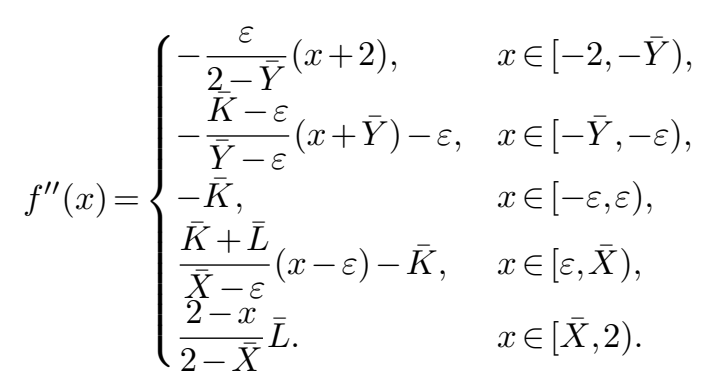

(ii) The first integration of the function $f^{\prime \prime}(x)$ with use of boundary conditions $f^{\prime}(-2)=f^{\prime}(2)=0$ yields

$$
f^{\prime}(x)= \begin{cases}-\frac{\varepsilon(2+x)^{2}}{4(1-\varepsilon)}, & x \in[-2,-2 \varepsilon), \\ \varepsilon(\varepsilon-1+x)+\frac{x^{2}}{2}-\bar{K}\left(2 \varepsilon+2 x-\frac{x^{2}}{2 \varepsilon}\right), & x \in[-2 \varepsilon,-\varepsilon), \\ -\frac{\varepsilon}{2}(2-\varepsilon)-\frac{\bar{K}}{2}(3 \varepsilon+2 x), & x \in[-\varepsilon, \varepsilon), \\ \frac{6 \varepsilon^{2}-2 \varepsilon^{4}+2 \varepsilon^{3} x+2 \varepsilon x^{2}-\varepsilon^{2}(2+x)^{2}}{2 \varepsilon(2-\varepsilon)}+ & \\ +\bar{K} \frac{8 \varepsilon^{3}-x \varepsilon(8-5 x)+2 x^{2}-\varepsilon^{2}(4+8 x)}{2 \varepsilon(2-\varepsilon)}, & x \in[\varepsilon, 2 \varepsilon), \\ -\frac{\varepsilon(2+6 \bar{K}-\varepsilon)(2-x)^{2}}{4(2-\varepsilon)(1-\varepsilon)}, & x \in[2 \varepsilon, 2),\end{cases}
$$

where $\bar{X}=\bar{Y}=2 \varepsilon$ and $\bar{K}, \bar{L}$ are chosen such that the boundary condition $f^{\prime}(2)=0$ is satisfied. Then

$$
\bar{L}=\varepsilon+\bar{K} \frac{\bar{X}+\bar{Y}+2 \varepsilon}{2-\varepsilon}=\varepsilon+\frac{6 \varepsilon}{2-\varepsilon} \bar{K} .
$$

The second integration with the use of boundary conditions $f(-2)=\bar{A}>0$ and $f(2)=$ $\bar{B}=\varepsilon>0$ yields

$$
f(x)= \begin{cases}\frac{\varepsilon\left(2+\varepsilon-3 \varepsilon^{2}\right)}{1-\varepsilon} \bar{K}+ & x \in[-2,-2 \varepsilon), \\ +\frac{\varepsilon\left(20-12 \varepsilon-28 \varepsilon^{2}+12 \varepsilon^{3}-12 x-6 x^{2}-x^{3}\right)}{12(1-\varepsilon)}, & x \in[-2 \varepsilon,-\varepsilon), \\ \frac{10 \varepsilon^{3}+12 \varepsilon^{2}(1-x)-6 \varepsilon x^{2}-x^{3}}{6 \varepsilon} \bar{K}+ & \\ +\frac{10 \varepsilon^{2}+4 \varepsilon^{3}-2 \varepsilon^{4}-6 \varepsilon^{2} x+6 \varepsilon^{3} x+3 \varepsilon^{2} x^{2}+\varepsilon x^{3}}{6 \varepsilon}, & x \in[-\varepsilon, \varepsilon), \\ \frac{1}{6} \bar{K}\left(11 \varepsilon^{2}+\varepsilon(12-9 x)-3 x^{2}\right)+ & \\ +\frac{1}{6}\left(10 \varepsilon+4 \varepsilon^{2}-3 \varepsilon^{3}-6 \varepsilon x+3 \varepsilon^{2} x\right), & x \in[\varepsilon, 2 \varepsilon), \\ \frac{-16 \varepsilon^{4}+8 \varepsilon^{3}(1+3 x)-12 \varepsilon^{2}\left(x^{2}+x-2\right)+\varepsilon x^{2}(12-5 x)-2 x^{3}}{6 \varepsilon(2-\varepsilon)} \bar{K}+ & \\ +\frac{4 \varepsilon^{5}-\varepsilon^{4}(12+6 x)+\varepsilon^{3}\left(-2+18 x+3 x^{2}\right)+\varepsilon^{2}\left(20-12 x-6 x^{2}-x^{3}\right)+2 \varepsilon x^{3}}{6 \varepsilon(2-\varepsilon)}, & x \in[2 \varepsilon, 2), \\ -\frac{\varepsilon(x-2)^{3}}{2(2-\varepsilon)(1-\varepsilon)} \bar{K}+ & \\ +\frac{\varepsilon\left[12 \varepsilon^{2}+\varepsilon\left(-44+12 x-6 x^{2}+x^{3}\right)+\left(40-24 x+12 x^{2}-2 x^{3}\right)\right]}{12(2-\varepsilon)(1-\varepsilon)}, & \end{cases}
$$


after substituting for $\bar{A}, \bar{B}, \bar{X}$, and $\bar{Y}$, where constants $\bar{A}$ and $\bar{B}$ are chosen to satisfy boundary conditions $f(-2)=\bar{A}$ and $f(2)=\bar{B}=\varepsilon$. This leads to a relationship between $\bar{A}$ and $\bar{B}$ :

$$
\begin{aligned}
\bar{A}= & \bar{B}+\frac{1}{6}\left[-2 \varepsilon^{3}+\bar{K}(2+\bar{Y})(\bar{X}+\bar{Y})+\varepsilon^{2}(2 \bar{K}-\bar{X}-\bar{Y})\right. \\
& +2 \varepsilon(4+\bar{X}+\bar{Y}+\bar{K}(2+\bar{X}+\bar{Y}))] \\
= & -\varepsilon^{3}+\left(\frac{4}{3}+3 \bar{K}\right) \varepsilon^{2}+\left(\frac{7}{3}+2 \bar{K}\right) \varepsilon
\end{aligned}
$$

(iii) The Lyapunov condition requires the coefficient $A_{2}$, defined in (4.7), to be negative. We plug $f, f^{\prime}$, and $f^{\prime \prime}$ into its formula to find conditions on remaining parameters $\bar{K}$ and $\varepsilon$ that guarantee $A_{2}\left(x, f, f^{\prime}, f^{\prime \prime}, \sigma\right)<0$. For $x \in[-\varepsilon, \varepsilon)$ we have

$$
\begin{aligned}
A_{2}(x, \sigma)= & \frac{3 \varepsilon^{3}}{3\left(1+x^{2}\right)}\left(x^{4}+x^{2}-2\right) \\
& -\frac{\varepsilon^{2}}{3\left(1+x^{2}\right)}\left[\left(2+x^{2}\right)\left(9 x^{3}+4 x^{2}+3 x-4+11 \bar{K}\left(x^{2}-1\right)\right)\right] \\
& +\frac{\varepsilon}{3\left(1+x^{2}\right)}\left[\left(2+x^{2}\right)\left(18 x^{3}-10 x^{2}+6 x+10+3 \bar{K}\left(9 x^{3}-4 x^{2}+3 x+4\right)\right)\right] \\
& +\frac{1}{3\left(1+x^{2}\right)}\left[3 \bar{K}\left(5 x^{6}+13 x^{4}+\left(6-\sigma^{2}\right) x^{2}-\sigma^{2}\right)\right] .
\end{aligned}
$$

For $\bar{K}=\frac{1}{\varepsilon}$ we obtain

$$
A_{2}(x, \sigma)=\left(8-\frac{\sigma^{2}}{\varepsilon}\right) \frac{1}{1+x^{2}}+\mathcal{O}(\varepsilon) .
$$

For small enough $\varepsilon>0$ the coefficient satisfies $A_{2}(x, \sigma)<-c<0$ for some $c \in \mathbb{R}$ and for $x \in[-\varepsilon, \varepsilon]$. Then the SLF condition is valid for all $x \in[-2,2]$ since

$$
\mathcal{L} V(x, y, \sigma) \sim A_{3}(x) y^{3}+A_{2}(x) y^{2}=-2(x y)^{2}\left|y f^{\prime}(x)\right|+A_{2}(x) y^{2} \rightarrow-\infty
$$

both for $|x|<\varepsilon\left(A_{3}(x) y \leq 0, A_{2}(x)<0\right)$ and for $|x|>\varepsilon\left(A_{3}(x) y<0\right)$ as $|y| \rightarrow \infty$. Moreover, the stochastic Lyapunov function connects in a $\mathscr{C}^{2}$ manner to $V(x, y, \sigma)=$ $\bar{A} \hat{V}(x, y)$ for $x \leq-2$ and to $V(x, y, \sigma)=\varepsilon \hat{V}(x, y)$ for $x \geq 2$. Note that for $y<-1$ we need to define $V(x, y, \sigma)=V(-x,-y, \sigma), V(x, y, \sigma)=\varepsilon \hat{V}(x, y)$ for $x \leq-2$, and $V(x, y, \sigma)=\bar{A} \hat{V}(x, y)$ for $x \geq 2$.

Below is the summary of all boundary conditions for $f(x, \sigma)$ in the upper half-strip $(|x| \leq 2, y \geq 1)$ :

$$
\begin{aligned}
f(-2) & =-\varepsilon^{3}+\frac{9 \bar{K}+4}{3} \varepsilon^{2}+\frac{6 \bar{K}+7}{3} \varepsilon, & f(2, \sigma) & =\varepsilon, \\
f^{\prime}(-2) & =0, & f^{\prime}(2) & =0, \\
f^{\prime \prime}(-2) & =0, & f^{\prime \prime}(2) & =0,
\end{aligned}
$$

and analogously by symmetry in the lower half-strip $(|x| \leq 2, y \leq-1)$.

(c) Final patching. Now we will smoothly patch $\bar{A} \hat{V}(x, y)$ and $\bar{B} \hat{V}(x, y)$ in the region $|y|<1$. This is done in a straightforward way by using a fifth-order polynomial as a smoothing function close to the $x$-axis. 
We define the function $V(x, y, \sigma)=V_{+}(x, y)$ on $|y|<1, x>2$ and $V(x, y, \sigma)=$ $V_{-}(x, y)$ on $|y|<1, x<-2$ separately. Let $V_{ \pm}(x, y)=g_{ \pm}(y) \hat{V}(x, y)$, where $g_{ \pm}(y)$ satisfy boundary conditions (smoothness requirements)

$$
\begin{aligned}
g_{+}(1) & =\varepsilon \quad \text { and } \quad g_{+}(-1)=\bar{A}, \\
g_{-}(1) & =\bar{A} \quad \text { and } \quad g_{-}(-1)=\varepsilon, \\
g_{ \pm}^{\prime}( \pm 1) & =0 \quad \text { and } \quad g_{ \pm}^{\prime \prime}( \pm 1)=0 .
\end{aligned}
$$

Polynomial functions of the lowest order that satisfy all stated requirements are

$$
\begin{aligned}
& g_{+}(y)=\bar{A}-(\bar{A}-\varepsilon) \frac{\int_{-1}^{y}(u+1)^{2}(u-1)^{2} d u}{\int_{-1}^{1}(u+1)^{2}(u-1)^{2} d u}, \\
& g_{-}(y)=\varepsilon+(\bar{A}-\varepsilon) \frac{\int_{-1}^{y}(u+1)^{2}(u-1)^{2} d u}{\int_{-1}^{1}(u+1)^{2}(u-1)^{2} d u} .
\end{aligned}
$$

One needs to make sure that $\mathcal{L} V(x, y, \sigma) \rightarrow-\infty$ while $y$ stays bounded as $|x| \rightarrow \infty$. The stochastic system (2.3)-(2.4) has the following approximate form in this region:

$$
\begin{aligned}
& d x=\left(-x^{3}+\mathcal{O}\left(x^{2} y\right)\right) d t+\sigma d \xi, \\
& d y=(-x+\mathcal{O}(1+y)) d t,
\end{aligned}
$$

yielding

$$
\mathcal{L} V(x, y, \sigma)=-2 x^{4} g_{ \pm}(y)+\delta\left(x^{3}, y, g_{ \pm}, g_{ \pm}^{\prime}\right),
$$

where $\delta\left(x^{3}, y, g_{ \pm}, g_{ \pm}^{\prime}\right)=\mathcal{O}\left(x^{3}\right)$ since $y, g_{ \pm}(y)$ and $g_{ \pm}^{\prime}(y)$ are all nonzero and bounded by a constant inside $|y|<1$. Since the leading term is negative (and grows to $-\infty$ as $|x| \rightarrow$

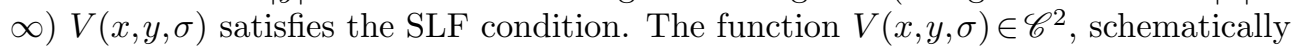
plotted on Figure 4.2, has the form

$$
V(x, y, \sigma)= \begin{cases}\varepsilon \hat{V}(x, y) & \text { if } x>2 \text { and } y>1, \\ \varepsilon \hat{V}(x, y) & \text { if } x<-2 \text { and } y<-1, \\ \bar{A} \hat{V}(x, y) & \text { if } x<-2 \text { and } y>1, \\ \bar{A} \hat{V}(x, y) & \text { if } x>2 \text { and } y<-1, \\ f_{\sigma}(x) \hat{V}(x, y) & \text { if }|x| \leq 2 \text { and } y \geq 1, \\ f_{\sigma}(-x) \hat{V}(x, y) & \text { if }|x| \leq 2 \text { and } y \leq-1, \\ g_{+}(y) \hat{V}(x, y) & \text { if } x \geq 2 \text { and }|y| \leq 1, \\ g_{-}(y) \hat{V}(x, y) & \text { if } x \leq-2 \text { and }|y| \leq 1, \\ \text { arbitrary } & \text { if }|x|<2 \text { and }|y|<1\end{cases}
$$

where $\hat{V}(x, y)=(x-y)^{2}+y^{2}, \bar{K}=\frac{1}{\varepsilon}$, and $\varepsilon$ is small enough. Note that the regularity of the process $(x(t), y(t))$ (condition (3.4)) is trivially satisfied for every initial condition. It is implied by a weaker SLF condition of the form $\mathcal{L} V \leq c V$ for some positive constant $c$ [11]. The process is therefore regular for all initial conditions.

Note that in order to satisfy $V(x, y, \sigma)>0$ for all $x, y \in \mathbb{R}$ we may add an arbitrary positive constant to the function $V$. The other properties of $\mathcal{L} V(x, y, \sigma)$ do not depend on the choice of this constant.

REMARK 4.1. Theorem 4.1 can be proven for a more general class of dynamical systems (2.5)-(2.6) as pointed out in Remark 2.1 with a minor change in the proof, i.e., the stochastic Lyapunov function will be based on $\hat{V}(x, y)=(a x-y)^{2}+y^{2}$ where $a$ is the parameter of the system. 


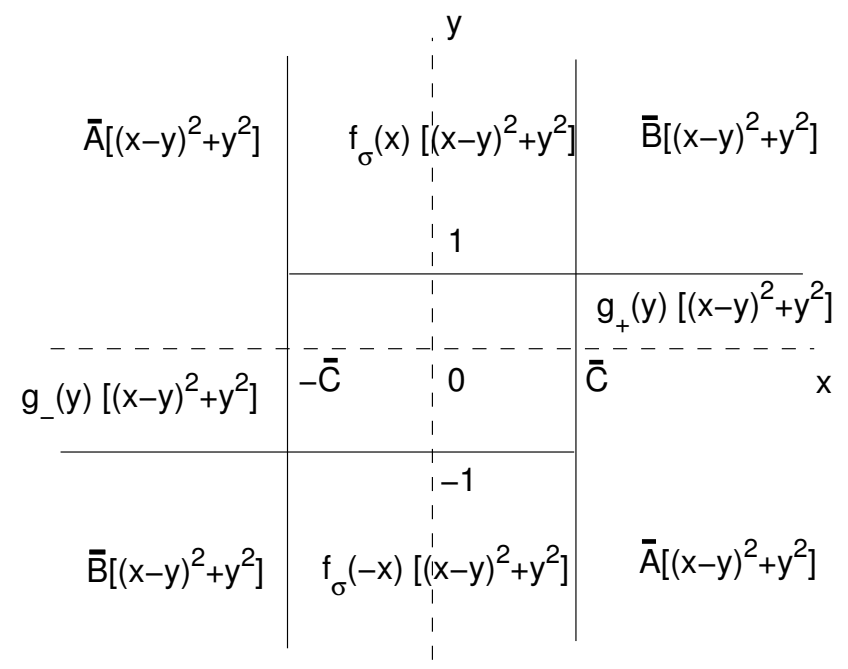

FIG. 4.2. A schematic picture of the stochastic Lyapunov function.

\section{First exit time problem}

The dynamics of (2.3)-(2.4) is very sensitive to noise in the slow region, i.e., along the $y$-axis, until the trajectory crosses the $x$-nullcline. However, in the rest of the phase space noise does not considerably alter the trajectory. Noise-driven collapses together with the system's resetting dynamical property force trajectories to repeatedly visit a small rectangle around the origin. Therefore we can say that the system has short memory, e.g. it effectively resets once it enters the neighborhood of origin even though the trajectory may travel very far away along the $y$-axis before it returns back towards the origin.

The time/location of collapses is studied as the first exit time problem from the noisy funnel region between the $y$-axis and the nullcline $y=x+4 / x$. Since the initial condition is quickly forgotten by the system we choose it to be fixed $(x, y)=(0,1)$ (thus restraining only to the upper half of $\mathbb{R}^{2}$ in the symmetric system). Alternatively, one may use a random initial condition (say, $y=1$ and $x \in[-4,4]$ according to a chosen distribution). The first exit time problem corresponding to (2.3)-(2.4) is

$$
\begin{cases}d x=-x\left(4-x y+x^{2}\right) d t+\sigma d W, & x \text {-dynamics } \\ d y=\frac{y-x^{3}}{1+x^{2}} d t, & y \text {-dynamics } \\ x(0)=0, \quad y(0)=1, & \text { initial conditions } \\ 0=4-x y+x^{2}, & \text { exit condition }\end{cases}
$$

The asymptotic regime of interest is $y \rightarrow \infty$ (i.e., $t \rightarrow \infty$ ) where $x \approx 0$. In this regime (2.3)-(2.4) is accurately approximated by

$$
\begin{aligned}
& d x=-x(4-x y) d t+\sigma d W \\
& d y=y d t
\end{aligned}
$$


Transformation of the problem to a constant boundary using $\bar{x}=x-4 e^{-t}$ yields

$$
\begin{cases}d \bar{x}=\left(4 \bar{x}+\bar{x}^{2} e^{t}+4 e^{-t}\right) d t+\sigma d W, & x \text {-dynamics } \\ \bar{x}(0)=-4, & \text { initial conditions } \\ \bar{x}(t)=0, & \text { exit condition. }\end{cases}
$$

5.1. Numerical study of the first exit time problem. The first exit time problem (5.1) can be studied using a standard Monte Carlo method (MC). However, we are interested in a long time limit where standard MC methods are extremely computationally expensive. This is because the funnel-shaped region between the $y$ axis and the nullcline $y=x+4 / x$ is narrowing at an exponential rate as time progresses and, with a set value of noise level $\sigma$, trajectories are progressively less likely to stay inside the region as time grows (also the step size must be finer as the region gets narrower). The probability that a trajectory stays in the region is very small at long times and the event is therefore called rare. There have been effective algorithms designed to simulate rare events in various applications, for example in finding the harmonic measure for critical percolation and Ising clusters [15]. Here we develop a new computationally effective method, inspired by [15], suitable for the first exit time problem (5.1).

The idea behind the method is to "recycle" otherwise irrelevant trajectories, therefore we will call it Monte Carlo method with Recycling (MCR). In terms of the implementation, MCR simultaneously keeps track of many trajectories. Once a trajectory hits the boundary of the region it is replaced by a different, randomly chosen trajectory from the sample. Consequently the survival probability $S(t)$ is updated to take the replacement into account and the process continues. The method is similar to [15].

We define the survival probability $S(t)$ as a probability that at time $t$ the trajectory is still inside of the half-funnel, i.e., $0 \leq y<x+4 / x$ with $x \leq 1$ or $x \leq 0$ with $y \geq 0$. Since the stochastic process $(x(t), y(t))$ is Markov the survival probability at time $t+\Delta t$ may be calculated from $S(t)$ and the probability of an exit in the time interval $(t, t+\Delta t]$ as

$$
\begin{aligned}
S(t+\Delta t) & =S(t) \cdot P[\text { exit in }(t, t+\Delta t]] \\
& =S(t) \frac{N-k}{N}
\end{aligned}
$$

where $k$ represents the number of exited trajectories during $(t, t+\Delta t]$. The value of $k$ is either zero or one if $\Delta t$ is sufficiently small. The numerical algorithm of the MCR is based on the rule (5.6) with replacements of the exiting trajectories by ones that are still inside of the region and thus keeping the same size of the sample with the same statistical properties. The MCR algorithm is:

$1^{\circ}$ Initialization: Start with a population of $N$ trajectories at $t^{(0)}=0$ with the same initial condition

$$
\left(x_{n}^{(0)}, y_{n}^{(0)}\right)=(x, 1)
$$

where $x=0$. Set the initial values of the survival probability $S(t)$ at time $t$ and the probability $P(t)$ of having hit the boundary by time $t$

$$
S\left(t^{(0)}\right)=1-P\left(t^{(0)}\right)=1 .
$$



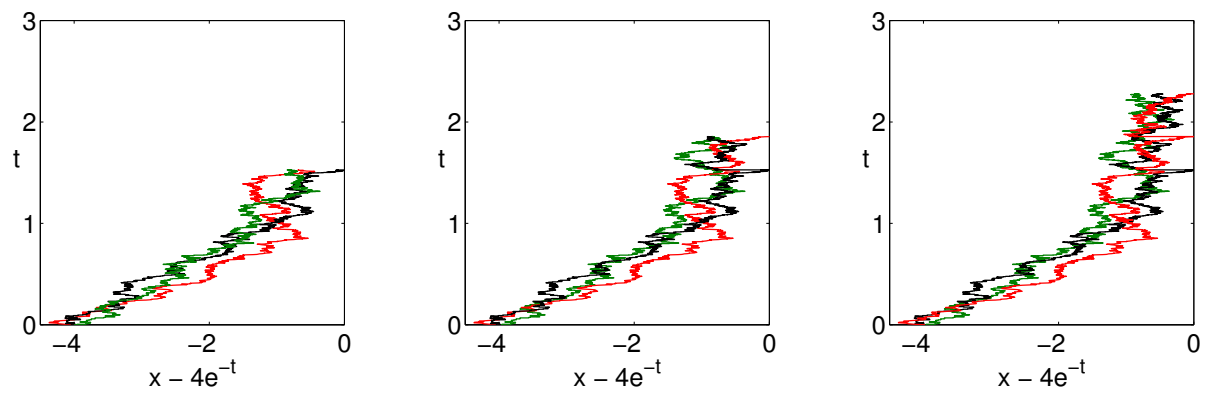

FIG. 5.1. The Monte Carlo method with recycling for three sample trajectories. Simulation is plotted at three different times: at the time of first, second and third hit of the boundary with $\sigma=1$. Each time a boundary is hit a trajectory is replaced by a different one from the surviving set.

$2^{\circ}$ Time step: For all trajectories $n \in\{1, \ldots, N\}$ apply the Euler scheme for (2.3)(2.4) to move forward in time.

$3^{\circ}$ Exit of trajectory: If $y_{n}^{(i)}>4-x_{n}^{(i)} y_{n}^{(i)}+x_{n}^{(i)^{2}}$ and $x_{n}^{(i)}>0$, replace trajectory $\left(x_{n}^{(i)}, y_{n}^{(i)}\right)$ by another, randomly chosen from the remaining $N-1$ trajectories. Update survival probability $S\left(t^{(i)}+\Delta t\right)=\frac{N-1}{N} S\left(t^{(i)}\right)$.

$4^{\circ}$ Loop: Repeat steps (2)-(3) until the terminal time.

By using the MCR algorithm we are able to compute survival probabilities of very small magnitudes. However, it is important to choose a number of sample trajectories $N$ large enough in order to visit a majority of possible outcomes at each time. Also, the time step needs to be adjusted in such a way that the probability of two trajectories exiting at the same time step is negligible. In practice we need to decrease the time step at the same rate as the nullcline approaches the $y$-axis. This happens at an exponential rate and the calculation therefore slows down exponentially in time. Figure 5.1 shows an illustration of the algorithm to the problem (5.1) for only three trajectories and three consequent hits of the boundary.

The algorithm is applied to (5.1) in order to provide information about the decay of the survival probability and the distribution of trajectories that have not hit the boundary yet. For the numerical analysis we choose terminal time $T=10$ and number of sample trajectories $N=2000$. We find that the numerical results for (5.1) and (5.4) are almost undistinguishable, particularly for large times (results not shown).

The survival probability is naturally a decreasing function of time and $y$. The simulation results on Figure 5.2 suggest that $\log |\log S(t)|$ as well as $\log m(t)$ are approaching a linear function for $t \rightarrow \infty$, where $m(t)=\mathbb{E}[\bar{x}(t)]$. In the case of $m(t)$ there is an initial transition from one to a different exponential rate of decay, as can be observed on the right panel of Figure 5.2. Results in both cases suggest that for different values of noise parameter $\sigma$ the slope is independent on $\sigma$ whereas the shift seems to depend linearly on some power of $\sigma$. We assume that $S(t)$ and $m(t)$ have the following description as $t \rightarrow \infty$ :

- The survival probability $S(t)$ has a double exponential asymptotic growth with appropriate $k, \delta$, and $\alpha$ :

$$
S(t) \sim e^{-K e^{\alpha t}}=e^{-k \sigma^{\delta} e^{\alpha t}} .
$$




\begin{tabular}{|l|c|c|c|c|c|c|}
\hline & $\alpha$ & $K$ & $B$ & $\beta$ & $C$ & $\gamma$ \\
\hline \hline$\sigma=1$ & 0.7185 & 0.7701 & 0.7984 & 0.3303 & 3.6095 & 1.5468 \\
\hline$\sigma=1 / 2$ & 0.7333 & 0.4220 & 0.4995 & 0.3290 & 4.0837 & 1.4020 \\
\hline$\sigma=1 / 4$ & 0.7591 & 0.2075 & 0.2750 & 0.3131 & 4.2989 & 1.2652 \\
\hline
\end{tabular}

TABLE 5.1. Fitted parameter values for the decay of $S(t)$ and $m(t)$.

- The mean $m(t)$ of a conditional distribution of $\bar{x}(t)$ asymptotically approaches an exponential function with appropriate $B=b \sigma^{\rho}, \beta$ and $C, \gamma>\beta$, where the prefactors $B$ and $C$ depend on the noise strength $\sigma$ :

$$
m(t)=B e^{-\beta t}+C e^{-\gamma t} \sim B e^{-\beta t}=b \sigma^{\rho} e^{-\beta t} .
$$
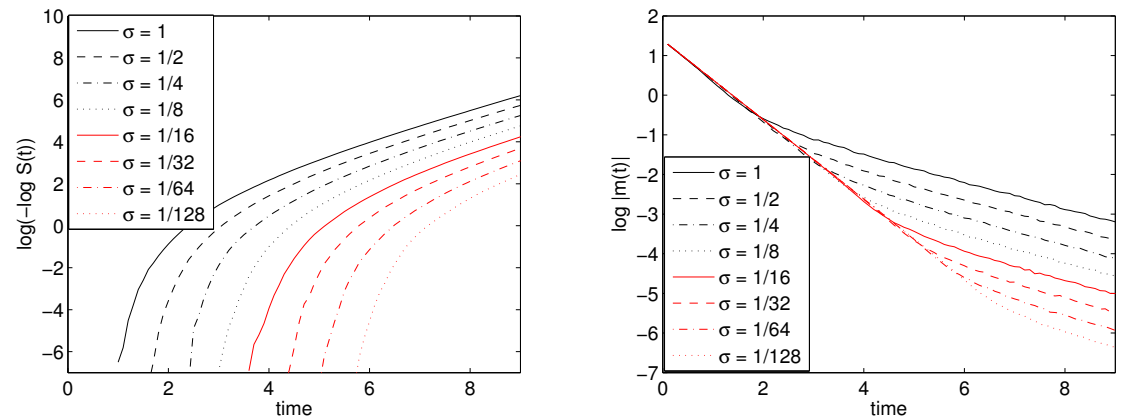

FIG. 5.2. (left) Survival probability $S(t)$ approaches a double exponential function as $t \rightarrow \infty$. This information is contained in the graph of $\log (-\log S(t))$ that appears to converge to a linear function for any $\sigma$. The slope of the function corresponds to $\alpha$ and the vertical shift corresponds to a linear function $\log k+\delta \log \sigma$. (right) The mean of the conditional distribution of $\bar{x}(t)$ as a function of time. The function can be accurately approximated by a sum of two exponential functions, the noise magnitude controls prefactors.

To estimate values of coefficients $\alpha, K, \delta$ and $\beta, B$ for different $\sigma$, we fit numerical data (for times $t_{i} \in\{0, \ldots, T\}$ ) to a function of the form (5.7) and (5.8). The best fit in $L^{2}$ space for this rule is a solution of two separate minimization problems

$$
\begin{gathered}
(\alpha, K)=\operatorname{argmin} \sum_{t_{i}=t_{i^{*}}}^{T}\left(\log \left(-\log S\left(t_{i}\right)\right)+\log K-\alpha t_{i}\right)^{2}, \\
(\beta, \gamma, B, C)=\operatorname{argmin} \sum_{t_{i}=0}^{T}\left(\log m\left(t_{i}\right)-\log \left(B e^{-\beta t_{i}}+C e^{-\gamma t_{i}}\right)\right)^{2} .
\end{gathered}
$$

Note that the fitting function in (5.9) is chosen to match the values only in the asymptotic regime, i.e., after some time (specified by index $i^{*}$ ). For $\sigma=1, \frac{1}{2}$, and $\frac{1}{4}$ the minimization problems yield parameter values in Table 5.1, where values of $b$, $\delta$, and $\rho$ may be found from $B$ a posteriori. Numerical results for coefficients $\beta$ and $\alpha$ seem to be roughly independent of the noise magnitude, implying the asymptotic form for $S(t)$ and $m(t)$, with $\alpha, \beta$ approximately constant and $\beta \approx 1 / 3$. 

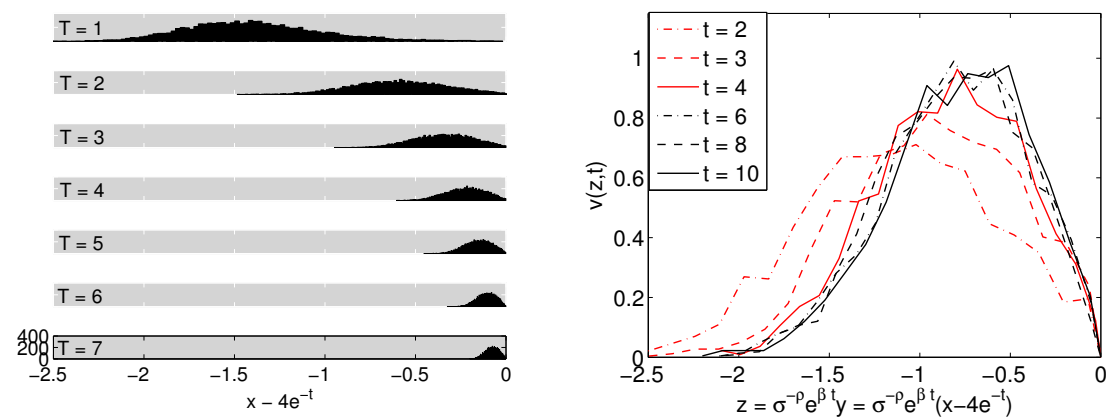

FIG. 5.3. (left) The histogram of trajectories in the MCR simulation at times $T=1,2, \ldots, 7$. The distribution is being squeezed towards the origin as time increases. (right) The rescaled probability density function $v(t, z)$ at times $T=2,3,4,6,8,10$. The functions converge to a time invariant pdf that captures behavior in the asymptotic regime $t=\infty$.

A natural way to confirm that (5.7) and (5.8) are true is to rescale the histograms on Figure 5.2 in the following way:

$$
\begin{gathered}
z=\sigma^{-\rho} e^{\beta t} \bar{x}, \\
u(t, \bar{x})=e^{-K e^{\alpha t}} v(t, z),
\end{gathered}
$$

where $u(t, \bar{x})$ is the unconditional pdf on $(-\infty, 0)$ and $v(t, z)$ is the conditional pdf after the normalization. If both hypotheses are correct then as time grows to infinity the density function $v(t, z)$ should converge to a self-similar solution. This is confirmed by Figure 5.3 in the case $\sigma=1$.

5.2. Analytical study of the first exit time problem. The first exit time problem (5.4) is mathematically studied using a Fokker-Planck equation (FPE). The conditional probability density function $u(t, x)$ that $x(t)$ has not yet reached the boundary satisfies the FPE with a moving boundary

$$
\begin{aligned}
u_{t} & =\left(\left(4 x-x^{2} e^{t}\right) u\right)_{x}+\frac{\sigma^{2}}{2} u_{x x}, \\
0 & =u\left(t, 4 e^{-t}\right) .
\end{aligned}
$$

The numerical results suggest that the following three transformations should be used: shifting to a constant boundary, stretching to accommodate (5.8), and scaling to fit (5.7). Including all three transformations, i.e., shifting, stretching, and scaling, we formulate an ansatz for the asymptotic form of $u(t, \bar{x})$ for $t \rightarrow \infty$ where $\bar{x}=x-4 e^{-t}$ :

$$
u(t, \bar{x})=e^{-K e^{\alpha t}} \sigma^{-\rho} e^{\beta t} v\left(t, \sigma^{-\rho} e^{\beta t} \bar{x}\right)=\sigma^{-\rho} e^{\beta t-K e^{\alpha t}} v\left(t, \sigma^{-\rho} e^{\beta t} \bar{x}\right) .
$$

Note that

$$
S(t)=\int_{-\infty}^{0} u(t, \bar{x}) d \bar{x}=e^{-K e^{\alpha t}} \int_{-\infty}^{0} \sigma^{-\rho} e^{\beta t} v\left(t, \sigma^{-\rho} e^{\beta t} \bar{x}\right) d \bar{x}=e^{-K e^{\alpha t}} \int_{-\infty}^{0} v(t, \hat{x}) d \hat{x} .
$$

Therefore $v(t, \bar{x})$ is the conditional probability density function of $\bar{x}$ in the noise dominated region (it is normalized to give $\int_{-\infty}^{0} v(t, \bar{x})=1$ ). If we denote $z=\sigma^{-\rho} e^{\beta t} \bar{x}$ then 
the FPE becomes

$$
\begin{aligned}
v_{t} & =\alpha K e^{\alpha t} v-\left(\left((4+\beta) z+z^{2} \sigma^{\rho} e^{(1-\beta) t}+4 \sigma^{-\rho} e^{(\beta-1) t}\right) v\right)_{z}+\frac{\sigma^{2(1-\rho)}}{2} e^{2 \beta t} v_{z z}, \\
0 & =v(t, 0) .
\end{aligned}
$$

For $t$ large we can use the numerical evidence that $\alpha, \beta \in(0,1)$ to obtain effective long time behavior as

$$
v_{t}=e^{\alpha t}(\alpha K v)-\sigma^{\rho} e^{(1-\beta) t}\left(z^{2} v\right)_{z}+e^{2 \beta t} \frac{\sigma^{2(1-\rho)}}{2} v_{z z} .
$$

If the stationary distribution exists, then it satisfies the ODE where the left hand side of (5.18) is replaced by 0 and right hand side only contains terms with the same exponential rates. The existence of invariant measure is then closely connected to the magnitude of growth constants $\alpha$ and $\beta$. It is not difficult to see that the only way this equation has nontrivial but normalizable solutions with boundary conditions $v(t, z=$ $0)=0$ and $\lim _{z \rightarrow-\infty} v(t, z)=0$ is when $\beta=\frac{1}{3}$ and $\alpha=\frac{2}{3}$. This is not inconsistent with numerically estimated values of constants $\alpha \approx 0.7185$ and $\beta=0.3303$. The problem becomes

$$
0=\lambda v-\sigma^{\rho}\left(z^{2} v\right)_{z}+\frac{\sigma^{2(1-\rho)}}{2} v_{z z}
$$

This is a linear eigenvalue problem with an eigenvalue $\lambda=\alpha K=\frac{2}{3} K$ that we can compute numerically for any value of $\rho$. We are interested in a positive normalizable solution - the eigenfunction (or the ground state) - corresponding to the smallest positive eigenvalue of the related operator. The eigenvalue problem (5.19) can be transformed to a singular, self-adjoint Sturm-Liouville (SL) problem on interval $(-\infty, 0]$ by an exponential transformation $v=w(z) \exp \left(\sigma^{3 \rho-2} z^{3} / 3\right)$, giving

$$
\lambda w=-\frac{\sigma^{2(1-\rho)}}{2} w_{z z}+\left(\frac{1}{2 \sigma^{2-4 \rho}} z^{4}+z \sigma^{\rho}\right) w,
$$

with $\lambda$ being the eigenvalue of the corresponding regular SL problem. Note that boundary conditions for the normalizable solution are

$$
w(0)=0 \quad \text { and } \quad w(-\infty)=0
$$

The functional dependence of the ground state eigenvalue on noise magnitude $\sigma$ can be found using a simple scaling argument. The linear transformation $z=\sigma^{2 / 3-\rho} \tilde{z}$ yields

$$
2 \lambda \sigma^{-2 / 3} w=-w_{\tilde{z} \tilde{z}}+\left(\tilde{z}^{4}+2 \tilde{z}\right) w .
$$

Since $2 \lambda \sigma^{-2 / 3}$ is an eigenvalue of the right-hand side operator that does not depend on a noise strength $\sigma$, the eigenvalue itself should be independent of $\sigma$. This implies $\lambda \sim \sigma^{2 / 3}$, i.e., $\delta=2 / 3$ in (5.7).

Next we turn our attention to an asymptotic calculation of the ground state eigenvalue using a WKB method. In order to turn the SL problem (5.20) into the right form, we rescale the independent variable $z$ and introduce a new variable $\bar{z}=$ $\left(2 \sigma^{2-4 \rho}\right)^{-\frac{1}{4}} z$. The result after dropping bars is

$$
\frac{\sigma}{\sqrt{8}} w_{z z}+(\lambda-V(z)) w=0
$$


where

$$
V(z)=z^{4}+\left(2 \sigma^{2}\right)^{\frac{1}{4}} z
$$

Let us denote

$$
\phi(z)=\lambda-V(z)=\lambda-\left(z^{4}+\left(2 \sigma^{2}\right)^{\frac{1}{4}} z\right) .
$$

The ODE we obtained has a form of a time-independent Schrödinger equation with a potential $V(z)$ for $z \leq 0$ and $V(z)=\infty$ for $z>0$ (this implies $w(0)=0$ ). The parameter $\sigma$ is small and as $\sigma \rightarrow 0$ the potential asymptotically approaches the potential of an anharmonic oscillator $V(z)=z^{4}$. The physical interpretation of the problem suggests that the smallest eigenvalue of the problem is positive. Otherwise there would need to be an exponentially growing pattern in the time evolution of the survival probability that contradicts its non-increasing property. Therefore we can apply the WKB approximation, discussed in J. D. Murray [14] to find an approximate solution of

$$
\varepsilon w_{z z}+\left[h_{0}(\varepsilon) \phi_{0}(z)+h_{1}(\varepsilon) \phi_{1}(z)\right] w=0,
$$

where $\varepsilon=\frac{\sigma}{\sqrt{8}}$ is the small parameter and

$$
\begin{aligned}
h_{0}(\varepsilon) & =1, & \phi_{0}(\varepsilon)=\lambda-z^{4}, \\
h_{1}(\varepsilon) & =2 \sqrt{\varepsilon}, & \phi_{1}(z)=-z .
\end{aligned}
$$

There are two distinct regions: $z \in(-\infty, \hat{z})$ where $\phi_{0}(z)<0$ and $(\hat{z}, 0)$ where $\phi_{0}(z)>0$. The constant $\hat{z}=-\lambda^{1 / 4}<0$ is the turning point, i.e., $\phi_{0}(\hat{z})=0$. We can approximate the solution of (5.26) in both regions. First, if $\phi_{0}(z)>0$ and $\sigma$ is small we use the asymptotic form as in [14]

$$
w_{+}(z) \sim \frac{K}{\phi_{0}(z)^{1 / 4}} \exp \left(i \frac{1}{\sqrt{\varepsilon}} \int_{z_{c}}^{z} \sqrt{\phi_{0}\left(z^{\prime}\right)} d z^{\prime}\right)=\frac{a}{\phi_{0}(z)^{\frac{1}{4}}} \sin U+\frac{b}{\phi_{0}(z)^{\frac{1}{4}}} \cos U,
$$

where

$$
U=\frac{1}{\sqrt{\varepsilon}} \int_{z_{c}}^{z} \sqrt{\phi_{0}\left(z^{\prime}\right)} d z^{\prime}
$$

and $z_{c} \in(-\infty, 0)$ is an arbitrary integration constant; we take $z_{c}=\hat{z}$. Second, if $\phi_{0}(z)<$ 0 the solution $w_{-}(z)$ is a combination of exponentially growing and decaying solutions

$$
w_{-}(z) \sim \frac{A}{\phi_{0}(z)^{1 / 4}} e^{U}+\frac{B}{\phi_{0}(z)^{1 / 4}} e^{-U} .
$$

In order to find eigenvalues of the problem we need to connect solutions (5.29) and (5.31) that are both singular at the turning point $\hat{z}$. This is done by locally solving (5.26) around $\hat{z}$. This matching process discussed in [14] yields a connection formula

$$
a=\frac{1}{\sqrt{2}}\left(\frac{B}{2}-A\right), \quad b=\frac{1}{\sqrt{2}}\left(\frac{B}{2}+A\right) .
$$

Required decay at $z=-\infty$ forces $B=0$, therefore $a=-b$. The boundary condition at $z=0$ then gives $\sin U=\cos U$, yielding $U=\frac{\pi}{4}+n \pi$. The quantization condition in terms of $\sigma$ therefore has a form

$$
\left(\frac{8}{\sigma^{2}}\right)^{1 / 4} \int_{\hat{z}}^{0} \sqrt{\lambda-z^{\prime}} d z^{\prime}=\frac{\pi}{4}+n \pi
$$




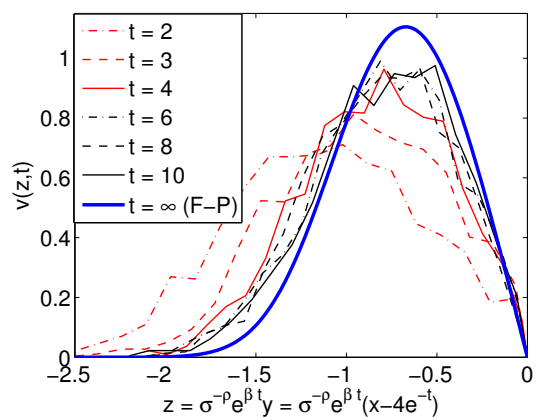

FIG. 5.4. The comparison of MCR and FPE solutions in a long-time limit. Thin lines are probability density functions of the conditional survival probability distribution inside the noise-sensitive region found by $M C R$ (for times $t=2, \ldots 10$ ), and the solid thick line is the numerically found and normalized eigenfunction of the stationary FPE corresponding to the first exit time problem. This line seems to capture the limiting distribution of the exit time problem.

The ground state corresponding to the smallest eigenvalue satisfies $n=0$ and it remains nonnegative for all $z \in(-\infty, 0]$. Since $\hat{z}=-\lambda^{1 / 4}$ we have

$$
\int_{-\lambda^{1 / 4}}^{0} \sqrt{\lambda-z^{\prime 4}} d z^{\prime}=\lambda^{3 / 4} \int_{-1}^{0} \sqrt{1-z^{\prime 4}} d z^{\prime}=\lambda^{3 / 4} \frac{\sqrt{\pi} \Gamma\left(\frac{1}{4}\right)}{8 \Gamma\left(\frac{7}{4}\right)} .
$$

The value of $\lambda$ can be found from (5.33):

$$
\lambda=\frac{\sigma^{2 / 3}}{2}\left[2 \sqrt{\pi} \frac{\Gamma\left(\frac{7}{4}\right)}{\Gamma\left(\frac{1}{4}\right)}\right]^{4 / 3} \propto \sigma^{2 / 3},
$$

where the scaling law is consistent with the intuitive answer found by the scaling argument $\lambda=\alpha K=\frac{2}{3} k \sigma^{\frac{2}{3}}$, yielding $\delta=2 / 3$.

We now test the agreement between the numerically found density function for the location of trajectories that have not yet hit the nullcline (MCR method) and the ground state, described by (5.19) on Figure 5.3. Note that (5.19) is a PDE with boundary conditions $v(0)=v(-\infty)=0$. The condition at $z=-\infty$ can be transformed to a first order boundary condition at zero by assuming that $\int_{-\infty}^{0} v(z) d z=1$. Then integration of the PDE results in

$$
0=\lambda \int_{-\infty}^{0} v+\left[-\sigma^{\rho} z^{2} v+\frac{\sigma^{2(1-\rho)}}{2} v_{z}\right]_{z} d z \quad \Rightarrow \quad v_{z}(0)=-\frac{2 \alpha k}{\sigma^{2(1-\rho)-\delta}}
$$

where $\alpha=\delta=2 / 3$. The results for the noise magnitude $\sigma=1$ are plotted on Figure 5.4. Table 5.2 shows parameter predictions by the numerical MCR method and by the analytic approach for $\sigma=1$. The value $k$ in the FPE approach is calculated from the approximate formula

$$
k=\frac{3}{2} \lambda \sigma^{-2 / 3} \approx \frac{3}{4}\left[\frac{2 \sqrt{\pi} \Gamma\left(\frac{7}{4}\right)}{\Gamma\left(\frac{1}{4}\right)}\right]^{4 / 3} \approx 0.6504 .
$$




\begin{tabular}{|l|c|c|c|}
\hline & $\alpha$ & $K$ & $\beta$ \\
\hline \hline Numerical method MCR & 0.7185 & 0.7701 & 0.3303 \\
\hline Analytical method FPE & $2 / 3$ & 0.6504 & $1 / 3$ \\
\hline
\end{tabular}

TABLE 5.2. Parameter values for different methods $(\sigma=1)$.

We remark that MCR method and analysis via the FPE give almost identical values for $\beta$ (exponential contraction rate on the horizontal axis) and similar values for $\alpha$ (double exponential decay rate of the survival probability) and K (the pre-factor).

As Figure 5.4 suggests, the solution of the FPE captures the trend in MCR results for $\sigma=1$ as time grows. Since the time $t=10$ is still not very large, the match is not perfect. Note that time $t=10$ in the MCR method corresponds to $y=e^{10} \approx 2.2 \cdot 10^{4}$, for which the time step required is the original time step reduced by this factor. The slowdown of the numerical calculation due to the exponential approach of the exit boundary is a major difficulty of any numerical method for this problem.

\section{Summary and discussion}

This paper discusses a planar dynamical system exhibiting exponential blow-up of trajectories in the absence of noise and persistent oscillations when arbitrarily small noise is applied. This is caused by two dynamical features of the model. First, the nonlinearities in the system determine a shrinking basin of attraction of the diverging solution. The dynamics in the $x$-direction in this region is dominated by noise while the rest of the phase space is relatively impervious to perturbations. Second, trajectories from anywhere outside of the attraction region return to a neighborhood of the origin. These two properties account for the existence of an invariant probability measure that is proven by an explicit construction of the stochastic Lyapunov function. An alternative model is presented that does not have a stochastic Lyapunov function because its trajectories do not reset back to the origin.

The typical size of the noise-induced oscillations depends on the noise strength: noise must force the trajectory out of the ever-narrowing basin of attraction to generate a collapse, and weaker noise makes the trajectory exit the region later. The problem is formulated as a first exit problem and is studied both numerically and analytically, the latter in the regime of small noise. The problem of inefficiency of standard Monte Carlo algorithms for numerical simulations is resolved by introducing a modified Monte Carlo with Recycling (MCR) method. Evidence is found that the survival probability and conditional mean behave like

$$
S(t) \sim e^{-K e^{\alpha t}}, \quad m(t) \sim B e^{-\beta t},
$$

with appropriate $\alpha, \beta$ and $K, B$. In the analytical approach the first exit problem is replaced by a Fokker-Planck equation. The ansatz (6.1) and a solvability argument for $t \rightarrow \infty$ are used to confirm that values of $\alpha$ and $\beta$ agree with the numerical results. Further WKB analysis allows us to determine the remaining parameters and to compare the asymptotic stationary probability density function of the trajectories near the deterministic heteroclinic connection to infinity with the numerically observed density function at large times found by MCR method. Again, the analysis predicts the density function accurately.

The original motivation for this study came from models in fluid mechanics where rigid walls confining - and often driving - the fluid are (primarily for computational 
and analytical convenience) replaced by periodic conditions to eliminate boundary layers. In various cases including thermal and thermohaline convection and even shear flows, such a change in boundary conditions introduces spurious "runaway" solutions that have no counterpart in the physically bounded flows [3]. Indeed, physically realizable boundary conditions result in uniformly bounded solutions $[5,4,6,1]$. While direct numerical simulations on the a priori unbounded systems are sometimes used to produce predictions for statistical features of the physically bounded models, it remains an open problem to prove the reliability of the results and their relation to the original models. The investigations presented here demonstrate robust and quantifiable features of such runaway dynamical systems, in particular their sensitivity to the noise that is inevitable in scientific computation.

Acknowledgement. We are grateful to J. Mattingly, L. Sander, R. Ziff and R. Kollár for helpful discussions. This work was supported in part by the U. S. National Science Foundation Awards DMS-0554587, PHY-0555324, PHY-0855335 and by the European Commission Marie Curie International Reintegration Grant PIRG04-GA2008-239429.

\section{REFERENCES}

[1] N.J. Balmforth, S.A. Ghadge, A. Kettapun, and S.D. Mandre, Bounds on double-diffusive convection, J. Fluid Mech., 569, 29-50, 2006.

[2] K. Bod'ová, Topics in Applied Stochastic Dynamics, PhD. Thesis, University of Michigan, Ann Arbor, Michigan, 2009.

[3] E. Calzavarini, C.R. Doering, J.D. Gibbon, D. Lohse, A. Tanabe, and F. Toschi, Exponentially growing solutions in homogeneous Rayleigh-Bénard convection, Physical Review E, 73, 035301, 2006.

[4] P. Constantin and C.R. Doering, Variational bounds on energy dissipation in incompressible flows. 2. Channel Flow, Physical Review E, 51, 3192-3198, 1995.

[5] C.R. Doering and P. Constantin, Variational bounds on energy dissipation in incompressible flows-Shear Flow, Physical Review E, 49, 4087-4099, 1994.

[6] C.R. Doering and P. Constantin, Variational bounds on energy dissipation in incompressible flows .3. Convection, Physical Review E, 53, 5957-5981, 1996.

[7] R. Erban, S.J. Chapman, I.G. Kevrekidis, and T. Vejchodsky, Analysis of a stochastic chemical system close to a sniper bifurcation of its mean-field model, SIAM J. Appl. Math., 70, 9841016, 2009.

[8] A.A. Faisal, L.P.J. Selen, and D.M. Wolpert, Noise in the nervous system, Nature Reviews in Neuroscience, 9, 292-303, 2008.

[9] W. Horsthemke and R. Lefever, Noise-Induced Transitions: Theory and Applications in Physics, Chemistry, and Biology, Springer-Verlag Berlin, 1983.

[10] D.W. Hughes and M.R.E. Proctor, A low-order model of the shear instability of convection: Chaos and the effect of noise, Nonlin., 1, 127-153, 1990.

[11] R.Z. Khasminskii, Stochastic Stability of Differential Equations, Sijthoff and Nordhoff, 1980.

[12] A.J. McKane and T.J. Newman, Predator-prey cycles from resonant amplification of demographic stochasticity, Physical Review Letters, 94, 218102, 2005.

[13] C.B. Muratov and E. Vanden-Eijnden, Noise-induced mixed-mode oscillations in a relaxation oscillator near the onset of a limit cycle, CHAOS, 18, 015111, 2008.

[14] J.D. Murray, Asymptotic Analysis, Springer, 1984.

[15] D.A. Adams, L.M. Sander and R.M. Ziff, Harmonic measure for percolation and ising clusters including rare events, Physical Review Letters, 101, 144102, 2008.

[16] M. Scheutzow, An integral inequality and its application to a problem of stabilization by noise, J. Math. Anal. Appl., 193, 200-208, 1995.

[17] M. Scheutzow, Stabilization and destabilization by noise in the plane, Stoch. Anal. Appl., 11, 97-113, 1993.

[18] D. Sigeti and W. Horsthemke, Pseudo-regular oscillations induced by external noise, J. Stat. Phys., 54, 1217-1222, 1989. 